\title{
Incoherent spatial filtering with a scanning heterodyne system
}

\author{
Guy Indebetouw and Ting-Chung Poon
}

\begin{abstract}
Imaging systems with two pupils in the receiving optics have been used to synthesize bipolar point spread functions in incoherent image processing. We describe a two-pupil system using a scanning illumination technique and compare its attributes with more conventional techniques. Experimental examples using two-pupil interaction and an acoustooptic frequency offset for the direct 2-D bandpass filtering of diffusely reflecting objects are presented.
\end{abstract}

\section{Introduction}

The last decade has seen an impressive reemergence of the field of incoherent optical processing. The motivations for this activity are that, when compared with their coherent counterparts, incoherent systems have a much better SNR (because of their redundancy) ${ }^{1}$ and can accommodate much simpler interfacing devices. The constraints imposed on the optical transfer function (OTF) of a conventional spatially incoherent system, ${ }^{2}$ which must have a real positive point spread function (PSF), are severe and preclude the direct implementation of the most useful linear operations (Wiener filtering, edge enhancement, differentiation, deblurring, etc.), which require bipolar PFSs.

A variety of techniques have been proposed and demonstrated in an effort to overcome this limitation by synthesizing an arbitrary complex PSF with the aid of two-pupil optical systems. These techniques have been classified and analyzed comparatively by Lohmann and Rhodes. ${ }^{3}$ Their most important characteristics are briefly reviewed in the next section to place the remainder of this paper in proper perspective.

Although the processing step in many of these systems is parallel in the sense that an output is obtained instantly as in a conventional imaging system, a sequential scanning of that output is often used (although it is not strictly necessary in all cases) either to perform

Both authors are with Virginia Polytechnic Institute \& State University, Blacksburg, Virginia 24061; G. Indebetouw is in the Physics Department and T. C. Poon is in the Department of Electrical Engineering.

Received 19 September 1984.

0003-6935/84/244571-04\$02.00/0.

(C) 1984 Optical Society of America. a difference electronically or to demodulate a signal. One might, therefore, imagine equivalent systems capable of performing the same operations at the same speed but in which the desired PSF, instead of being synthesized in the receiving optical system, would be carried by the illumination and scanned over the input. In this mode of operation, the system's complexity is transposed from the receiving optics to the illumination. As we try to show in Sec. III, implementation of this type of system is often technically much simpler. One limitation of active illumination techniques is that they cannot easily deal with self-luminous inputs such as a CRT or a TV monitor. They can nevertheless accommodate any transparent or reflecting, specular, or diffuse inputs such as those most often encountered in machine vision and robotics. Here the advantage of a robust system with efficient illumination and ambient light rejection might not be negligible.

The last section of this paper gives some experimental results on the 2-D edge enhancement of diffusely reflecting objects. The incoherent bandpass filtering operation required was achieved with a two-pupil interaction scheme ${ }^{3}$ using a temporal frequency offset obtained acoustooptically. 4

\section{Two-Pupil Receiving Optics}

The methods reviewed by Lohmann and Rhodes ${ }^{3}$ use an optical system with two pupils to synthesize bipolar PSFs. (Arbitrary complex PSFs can be dealt with in a multichannel system.) There are two classes of methods, the direct difference methods and the pupil interaction methods. The first class synthesizes PSFs of the form

$$
\operatorname{PSF}=\left|p_{1}(x)\right|^{2}-\left|p_{2}(x)\right|^{2}
$$

by making a direct difference of two images obtained with the pupils $P_{1}(u)$ and $P_{2}(u)$, respectively. (Upper case letters are Fourier transforms of corresponding 
lower case letters.) In general, the pupils can be computer generated holograms. ${ }^{5,6}$

There are three possible ways of doing the difference. The most direct, a simultaneous electronic difference of two signals, requires synchronous scans of the two outputs of a dual channel system. This seems to be technologically difficult if not impractical. For stationary input, the two outputs can be obtained sequentially, stored, and digitally subtracted. ${ }^{7}$ Finally, the real time difference of two images can be achieved with a spatial carrier technique followed by a scan and electronic demodulation. The shifted carriers can be produced by interlaced gratings ${ }^{8-10}$ or by polarization coding. ${ }^{11,12}$ A major practical difficulty in the direct difference method is that the two channels that must be subtracted must be kept precisely balanced.

The methods of the second class use two-pupil interaction $^{3}$ and synthesize PSFs of the form

$$
\mathrm{PSF}=\operatorname{Re}\left[p_{1}(x) p_{2}^{*}(x)\right] .
$$

There are two possible approaches in synthesizing a PSF of this form. The first consists of combining the two pupils interferometrically and subtracting two outputs resulting from imaging with the combined pupils $P_{1}(u)+P_{2}(u)$ and $P_{1}(u)+\exp (i \pi) P_{2}(u)$, respectively. ${ }^{13,14}$ The second approach uses carrier techniques to offset the cross product term in the squared sum of the two impulse responses $p_{1}(x)$ and $p_{2}(x)$. This term can then be extracted by demodulation or filtering. Spatial carriers have been used to effect the offset. ${ }^{15-17}$ The spatial frequency offset is produced by combining the two pupils in a wavefront division interferometer, consequently taxing the spatial bandwidth requirement of the system. The output is then scanned and filtered electronically. Other methods use a temporal carrier ${ }^{18-20}$ and combine the pupils in an amplitude division interferometer. ${ }^{21}$ This has the advantage of relaxing the spatial bandwidth requirement and providing a local oscillator for precise heterodyning. Many of these techniques perform the optical processing steps instantly but produce an output from which the desired processed image must be extracted by filtering or demodulation. In principle, the spatial or temporal differentiating properties of some spatial light modulators ${ }^{22,23}$ could be used to demodulate the output in parallel. This, however, would transpose to the detection the difficulty in using such elements as an input device. Avoidance of such devices is one of the motivations in developing direct incoherent processing techniques. The most convenient and practical way to demodulate an image on a spatial or temporal carrier is to scan it and filter the temporal signal electronically.

\section{Two-Pupil Scanning Illumination Systems}

Equivalent processing methods, using the synthesis of bipolar PSFs with two pupils, can be achieved with systems where the PSFs are synthesized in the illumination setup and directly scanned over the input ${ }^{4}$ rather than synthesized in the receiving optics. In such scanning systems, the convolution is performed by the spatial shift provided by the scan and the spatial integration of the detector. This entirely eliminates the need for receiving optics and in some implementations can relax the stability and precision requirements of the system.

In principle, the methods described in the previous section can be implemented with scanning illumination. The two most appropriate examples will be discussed: the direct difference of the outputs of a two-channel system and the two-pupil interactions with temporal frequency offset. Examples of applications of the first technique have been published, ${ }^{24}$ and the second is demonstrated experimentally in the next section. In addition, we will first mention a single-pupil technique which is specific to the scanning illumination method.

If the input is a transparency scanned with an amplitude distribution $p(x)$, the electrical output of a pinhole detector in the far field is the square modulus of the convolution of $p(x)$ with the input amplitude transmittance. ${ }^{24,25}$ This is equivalent to the quadratic detector output of a coherent imaging system. The method is sensitive to input phase and, therefore, cannot be used with diffuse objects.

The same scanning system with a spatially integrating detector produces an output equivalent to that of an incoherent imaging system, namely, the convolution of $|p(x)|^{2}$ with the input intensity transmittance or reflectance. ${ }^{24}$ It is, therefore, very easy to perform the direct difference of two such channels on-line and with standard electronics to synthesize PSFs of the form given by Eq. (1). In a particular implementation of this technique, ${ }^{26}$ the two PSFs are projected collinearly and scanned over the input simultaneously. The two channels are subsequently separated on the basis of different polarizations or wavelengths, detected, and simultaneously subtracted electronically. Compared with the synchronous scans of two different outputs required by other methods, this is trivial to implement.

The PSFs of the form given by Eq. (2) can also be synthesized by two-pupil interaction in the scanning illumination mode. 4 Since a laser beam is most conveniently used to project the PSF onto the input, the pupils can, if necessary, consist of computer generated holograms producing the PSFs by diffraction. If it proves to be simpler, the PSFs can also be constructed directly with masks instead of the pupils. This adds some flexibility to the method. Furthermore, it becomes possible to use simple and reliable methods such as Bragg scattering on a traveling acoustic wave to produce a temporal frequency offset. ${ }^{4}$ This allows for high frequency shifts $(\sim 50 \mathrm{MHz})$ and consequently a very high scan rate as well as a stable efficient heterodyne detection. The problems of bias and pupil efficiencies are of the same nature as for the other multipupil methods. ${ }^{3,27}$ 


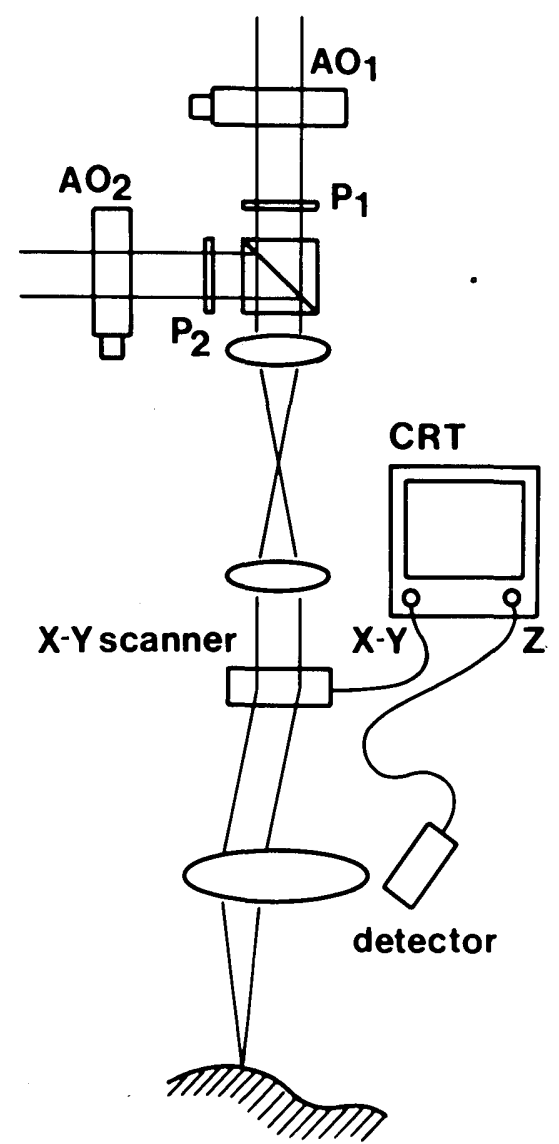

Fig. 1. Setup used for the two-pupil interaction synthesis of bipolar PSF with scanning illumination. AOs are Bragg acoustooptic cells. The transmitted beam is the diffracted phase shifted beam. The heterodyne signal from the detector modulates the CRT's beam intensity.

\section{Experimental Results}

As a demonstration of the last method mentioned in the previous section, we have applied the technique to the 2-D edge enhancement of diffusely reflecting objects by incoherent bandpass filtering. The system, sketched in Fig. 1, uses two mutually coherent laser beams to illuminate the pupils $P_{1}(u)$ (an annular aperture) and $P_{2}(u)$ (a small on-axis aperture). The two beams are frequency shifted by $80 \mathrm{kHz}$, the difference between two acoustooptic modulators working each near $40 \mathrm{MHz}$. The only reason for not working with a higher frequency shift was the lack of fast electronic equipment. An $x-y$ mirror scanner is used to deflect the collinear beams onto the input. The instantaneous signal detected by a photomultiplier gathering the reflected light is of the form

$$
\begin{aligned}
S(t)= & \iint \mid p_{1}\left(x-x^{\prime}, y-y^{\prime}\right) \exp \left(i \omega_{1} t\right) \\
& +\left.p_{2}\left(x-x^{\prime}, y-y^{\prime}\right) \exp \left(i \omega_{2} t\right)\right|^{2} R(x, y) d x d y,
\end{aligned}
$$

where $x^{\prime}, y^{\prime}=x^{\prime}(t), y^{\prime}(t)$ is defined by the scanner's motion, $\omega_{1}-\omega_{2}=\Omega=80 \mathrm{kHz}$, and $R(x, y)$ is the intensity reflectance of the input. The heterodyne signal at $\Omega$ can, therefore, synthesize an arbitrary bipolar PSF of the form given by Eq. (2). With the pupils used, this is the PSF corresponding to a 2-D bandpass filter.

Some results are shown in Figs. 2 and 3 for black let-
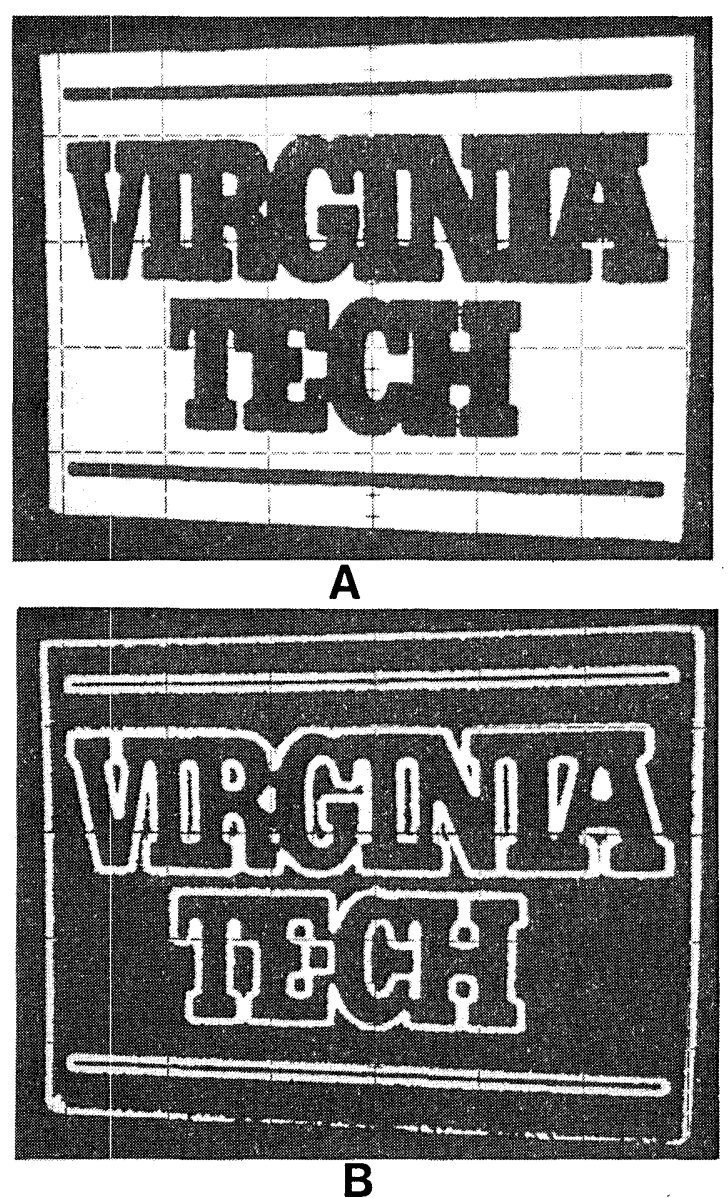

Fig. 2. Lowpass (A) and bandpass (B) filtered images of a black text on matte white background.

ters on matt white paper and for a small 3-D toy, respectively. In these pictures, the edge enhanced or bandpass filtered image is compared with a lowpass version of the same object. One of the pupils was a circular aperture of $\sim 1-\mathrm{mm}$ diameter, the other had an outside diameter of $\sim 2 \mathrm{~mm}$ and, for the bandpass image only, a circular central obstruction of $\sim 1-\mathrm{mm}$ diameter. With the $50-\mathrm{cm}$ focal length projection lens, this corresponds to a cutoff frequency of $\sim 6$ lines $/ \mathrm{mm}$ for both images and a mean bandpass frequency of $\sim 3$ lines $/ \mathrm{mm}$. The scanning rate was limited by the inertia of the galvanometer scanner to $\sim 1 \mathrm{sec} /$ frame for a $\sim 6-\times 6$-cm field with 150 lines. The output of the detector, after electronic bandpass filtering, is an ac signal at the heterodyne frequency of $80 \mathrm{kHz}$. For the present demonstration, this signal was used directly to modulate the CRT display's intensity. An envelope detector would obviously produce a more exact result, but we relied on the fact that the display's modulation does not respond to negative signals and that the display could not spatially resolve the $80-\mathrm{kHz}$ flicker.

\section{v. Conclusions}

We have demonstrated a two-pupil interaction method to synthesize bipolar PSFs for incoherent optical processing. The method is implemented by simultaneously scanning the input with the impulse responses corresponding to each pupil and by introducing, 


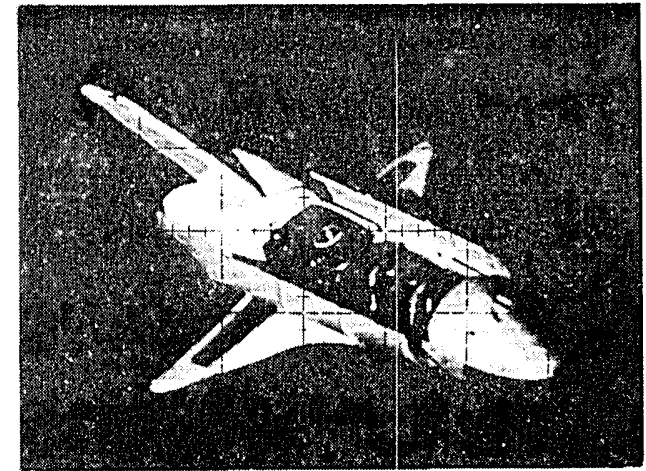

A

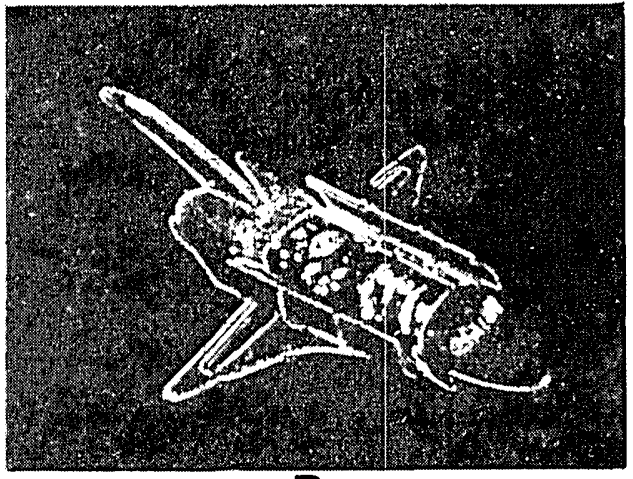

B

Fig. 3. Lowpass (A) and bandpass (B) filtered images of a small diffusely reflecting toy.

with the aid of an acoustooptic cell, a temporal frequency offset between the two pupils. Subsequent heterodyne detection demodulates the synthesized PSF.

We have tried to place this work in perspective by comparing the scanning illumination approach with other methods using two pupils in the receiving optics. Eliminating the receiving optics and producing the PSF in the illuminating beam seem to offer certain advantages of robustness and ease of implementation, which could be useful in applications such as robotics or machine vision.

\section{References}

1. P. Chavel and S. Lowenthal, "Noise and Coherence in Optical Image Processing. I. The Callier Effect and its Influence on Image Contrast," J. Opt. Soc. Am. 68, 559 (1978); "Noise and Coherence in Optical Image Processing. II. Noise Fluctuations," J. Opt. Soc. Am. 68, 721 (1978).

2. W. Lukosz, "Properties of Linear Low Pass Filters for Non-negative Signals," J. Opt. Soc. Am. 52, 827 (1962).

3. A. W. Lohmann and W. T. Rhodes, "Two-Pupil Synthesis of Optical Transfer Functions," Appl. Opt. 17, 1141 (1978).

4. T. C. Poon and A. Korpel, "Optical Transfer Function of an Acousto-optic Heterodyning Image Processor," Opt. Lett. 4, 317 (1979).
5. P. Chavel and S. Lowenthal, "Incoherent Optical-Image Processing Using Synthetic Holograms," J. Opt. Soc. Am. 66, 14 (1976).

6. B. Braunecker and R. Hauk, "Grey Level on Axis Computer Holograms for Incoherent Image Processing," Opt. Commun. 20, 234 (1977).

7. H. Bartelt and A. W. Lohmann, "Optical Processing of OneDimensional Signals," Opt. Commun. 42, 87 (1982).

8. S. R. Dashiell, A. W. Lohmann, and J. D. Michaelson, "Real Time Incoherent Optical-Electronic Image Subtraction," Opt. Commun. 8, 105 (1973).

9. G. Hausler and A. Lohmann, "Hybrid Image Processing," in Applications of Holography and Optical Data Processing, E. Marom, A. Friesem, and E. Wiener-Avnear, Eds. (Pergamon, New York, 1977), p. 9-18.

10. E. Marom and N. Konforti, "Low Frequency De-emphasis of the Modulation Transfer Function," Opt. Commun. 41, 388 (1982).

11. S. R. Dashiell and A. W. Lohmann, "Image Subtraction by Polarization-shifted Periodic Carrier," Opt. Commun. 8, 100 (1973).

12. G. Indebetouw, L. Bernardo, and M. Miller, "Real-time Incoherent Subtraction of Irradiance," Appl. Opt. 19, 1218 (1980).

13. W. T. Rhodes, "Phase-Switching Synthesis of Arbitrary Optical Transfer Functions," in Proceedings, 1976 International Optical Computing Conference, Capri, Italy, S. Horwitz, Ed. (IEEE, New York, 1977), pp. 62-64.

14. D. Gorlitz and F. Lanzl, "Methods of Zero-order Non-coherent Filtering," Opt. Commun. 20, 68 (1977).

15. A. W. Lohmann, "Incoherent Optical Processing of Complex Data," Appl. Opt. 16, 261 (1977).

16. W. Stoner, "Edge Enhancement with Incoherent Optics," Appl. Opt. 16, 1451 (1977).

17. W. Stoner, "Incoherent Optical Processing via Spatially Offset Pupil Masks," Appl. Opt. 17, 2454 (1978).

18. G. L. Rogers, "Non-coherent Optical Processing," Opt. Laser Technol. 8, 153 (1975).

19. A. Macovski, "Use of Frequency Offset in Incoherent Optical Data Processing," Appl. Opt. 12, 1745 (1973).

20. G. L. Rogers, "Temporal Modulation in Noncoherent Optical Data Processing," Appl. Opt. 13,997 (1974).

21. W. T. Rhodes, "Bipolar Pointspread Function Synthesis by Phase Switching," Appl. Opt. 16, 265 (1977).

22. H. H. Barrett, A. F. Gmitro, and M. Y. Chiu, "Use of an Image Orthicon as an Array of Lock-in Amplifiers," Opt. Lett. 6, 1 (1981).

23. A. S. Vasiliev, I. N. Kompanets, and A. V. Parfenov, "Advances in Development and Applications of Optically Controlled Liquid Crystal Spatial Light Modulators," Optik 67, 223 (1984).

24. G. Indebetouw, "Scanning Optical Data Processor," Opt. Laser Technol. 8, 197 (1981).

25. D. Kermish, "Partial Coherent Image Processing by Laser Scanning," J. Opt. Soc. Am. 65, 887 (1975).

26. G. Indebetouw, "Non-linear/adaptive Image Processing with a Scanning Optical System," Opt. Eng. 23, 73 (1984).

27. J. N. Mait and W. T. Rhodes, "Iterative Design of Pupil Functions for Bipolar Incoherent Spatial Filtering," Proc. Soc. Photo-Opt. Instrum. Eng. 292, 66 (1981).

Each of us wishes to gratefully acknowledge the financial support of the National Science Foundation for the research that lead to this report (grant ECS 8116337 for G.I. and grant ECS 8400635 for T.C.P.). We would also like to thank W. T. Rhodes for reading the manuscript and suggesting some improvements. 